\title{
Cognitive gains from video game use in older age: a review of the literature corroborating them
}

\begin{abstract}
This article contains succinct summaries of the published literature regarding strategies that could lead to improved cognitive functioning among older individuals, mechanisms explaining the positive psychological effects of video game use in older age, and psychological factors related to using video games in older age. Factors contributing to the positive impact of video game usage on older adults are discussed, followed by an explanation of how skills acquisition among older adults during video game training appears to transfer to non-practiced tasks, as this could create several advantages in their lives. Due to space limitations, this review does not cover all published literature in this area. Instead, we have focused mainly on the available evidence in support of video game use among older adults. A plethora of research indicates that benefits derived from a singular video game may generalize to a full genre of games. As technology continues to advance, researchers must explore new methods to improve cognitive functioning. Given the high incidence of cognitive impairment among older adults, the need for future research in this area is emphasized.
\end{abstract}

Volume 2 Issue I - 2018

\author{
Luciana Lagana ',' Giovanni Sosa, ${ }^{2}$ Marina \\ Zaher Nakhla,' 'Dillion Toscano' \\ 'Department of Psychology, California State University \\ Northridge, USA, CA \\ ${ }^{2}$ Office of Institutional Research, Chaffey College, USA, CA
}

Correspondence: Luciana Lagana ', Department of

Psychology, California State University Northridge, USA, CA, Tel +|8186772827, Email Luciana.Lagana@csun.edu

Received: November 22, 2017 | Published: January 19, 2018

Keywords: cognitive improvements, cognitive gains, cognitive training, cognitive function, aging, older adults, video game activities

\section{Introduction}

In this review article, the authors have summarized the published literature supporting the value of video game use on the following topics: improvement of cognitive functioning in older individuals, potential reasons for the positive effects of video game use in older age, and psychological factors related to using video games in older age. It is important for geriatric researchers and practitioners to identify approaches and interventions that minimize the negative effects of the various changes that occur within the aging body. Generally speaking, biological aging results in a decline of both physical and cognitive functioning. ${ }^{1-3}$ However, a growing body of literature indicates that taking part in physically and/or mentally stimulating activities may contribute to the maintenance of cognitive abilities and even lead to acquiring cognitive gains. ${ }^{4}$ It is important to identify ways to induce cognitive improvements in older age, especially considering that the population of the United States (U.S.) is aging rapidly, with the number of people age 65 and older expected to increase to almost 84 million by $2050 .{ }^{5}$ This suggests that there will likely be a rapid escalation in the number of older individuals living with age-related cognitive impairment. It is currently estimated that there are 5.5 million people in the U.S. who have been diagnosed with Alzheimer's disease, ${ }^{6}$ which is one of the most common forms of dementia. ${ }^{7}$ Thus, research aimed at helping older adults maintain good cognitive functioning is highly needed. Due to space limitations, this article is not meant to include all of the available research in this area; it contains mainly supporting evidence on the effects of video game use among older adults. Some opposing evidence is briefly mentioned when covering whether the skills acquired during video game training transfer to non-practiced tasks (which is a particularly controversial topic with ample mixed evidence).

\section{Age-related cognitive impairment}

"Normal" age-related cognitive impairment is characterized by problems in distractibility and executive functioning ${ }^{8}$ as well as in perception, memory, processing speed, attention, and motor function. ${ }^{9,10}$ Even slight levels of impairment in these cognitive domains can negatively impact psychological functioning. ${ }^{11}$ Dementia, a more serious form of cognitive impairment, is associated with various psychological symptoms of anxiety and depression (with about $43 \%$ of patients with dementia experiencing depression ${ }^{12}$ ) as well as with psychosis and apathy. ${ }^{12,13}$ According to the National Health Interview Survey, ${ }^{14}$ mild or moderate cognitive impairment is prevalent in approximately $4 \%$ of adults age 65 to 74 , specifically those who are not institutionalized. However, prevalence rates are higher with increasing age, with mild or moderate cognitive impairment occurring in about $10 \%$ of individuals age 75 to 84 , and in approximately $20 \%$ of people age 85 and older.

\section{Non-video game-based activities and older adults' cognitive functioning}

Although video game-based interventions are the focus of this article, it is important to briefly point out the results of research conducted on other kinds of cognitively stimulating activities, considering that the findings of several published studies have revealed that a variety of non-video game-based activities can help older adults' cognitive functioning. The common "use it or lose it" notion supports the view that engaging in a variety of activities can prevent or lessen age-related cognitive decline. For example, researchers found that approximately 30 to $40 \%$ of adults age 65 and older report being able to keep their memory sharp by participating in mentally stimulating activities such as reading as well as playing music or chess. ${ }^{15,16}$ Cognitive decline can also be reduced through engagement in physical activities and leisure mental activities. ${ }^{17}$

The available evidence indicates the existence of several beneficial effects stemming from participating in mentally stimulating activities in older age. For instance, when exploring the effects of a 10-session memory training program on older adults over a 5-year period in a randomized controlled study, significant improvements in memory 
abilities were found, which remarkably persisted after the 5-year period. ${ }^{18}$ Furthermore, a meta-analysis revealed that cognitive training significantly improves performance on various measures of executive as well as cognitive functioning. ${ }^{15}$ Similarly, engaging in mentally stimulating activities (e.g., playing piano, acting, and helping children with reading difficulties) can lead to significantly enhanced performance on memory components such as face-name recall and immediate recall, as well as on subjective measures of cognitive performance. ${ }^{15}$ While correlational in nature - it is also possible that those who possess greater levels of cognitive functioning already engage in a greater number of mental activities - the abovementioned findings suggest a meaningful relationship between mentally stimulating activities and cognitive functioning. The following paragraphs contain a summary of the available literature on engagement in video game activities in older age in relation to its multiple psychological and cognitive outcomes.

\section{The efficacy of video games use in older age: cognitive outcomes}

As technological advances occur at an accelerated rate, researchers must investigate techniques that could improve quality of life among typically vulnerable populations such as older adults. As previously mentioned, older adults living with cognitive impairments are more likely to experience depression. ${ }^{12-19}$ Using video games appears to enhance psychological outcomes in older age; these improvements may be attributable, at least partially, to the fact that game training increases motivation and addresses isolation. ${ }^{20}$ Video game designs and technologies have viable applications beyond just mere entertainment, as they can be utilized as teaching tools to improve patients' health outcomes and advance treatment. ${ }^{21}$ During the initial phases of video game research, a common research drawback was that most of the video games used in interventions were not readily available to the public; this limited the generalizability of the related research findings. However, this limitation is no longer as applicable as it was in the past, considering that an increasing amount of research investigating the benefits of using video games in older age is being conducted on publicly available video games.

Researchers examining differences among 140 older adults (age 63 to 92) who played digital games - relative to those who did not - measured two aspects of play: frequency (e.g., regular gamers, occasional gamers, and non-gamers) and type (e.g., digital card games, Wii games, and puzzle games). Older individuals who regularly or even occasionally engaged in digital games obtained significantly better scores on well - being in addition to lower scores on negative affect and depression. ${ }^{22}$ Moreover, in an experimental study regarding the effects of video games on older adults' affect, participants who exhibited symptoms of sub-syndromal depression were instructed to play Nintendo's Wii sports gaming console for a 12-week intervention period: 3 sessions lasting 35 minutes per week. ${ }^{19}$ Playing games that combined game play with exercise (referred to as exergames) enhanced cognitive stimulation, positive effect, and physical activity. A systematic review synthesized 38 studies on the impact of video gaming on health-related outcomes: remarkably, regardless of age, video games use improved $69 \%$ of psychological therapy outcomes and $50 \%$ of physical activity outcomes. ${ }^{23}$

\section{The efficacy of video games: cognitive outcomes}

Not only could video games have a positive effect on psychological health in older age, but, they could also have a similar effect on cognitive functioning. This is, based on the findings of a growing body of literature demonstrating the positive relationship between video game use and older adults' cognitive performance. ${ }^{24-28}$ For instance, older adults playing the commercially available video game Brain Age for 6 weeks experienced significant gains on a variety of cognitive outcomes compared to a control group (average $d=1.16$ ). ${ }^{29}$ Similar findings were reported on a sample of 22 non-institutionalized older individuals living in Holland who engaged in home-based training for the duration of 5 weeks (weekly amount of training time $=5$ hours). More specifically, their performance on Card Sorting Tasks [30] - a task of cognitive processing speed - was significantly improved. ${ }^{31}$

In an investigation conducted in Spain, engaging in computer games specifically constructed to improve cognitive rehabilitation ${ }^{32}$ resulted in decreased cognitive impairment on the Mini-Mental State Exam (MMSE), ${ }^{33}$ a neuropsychological assessment utilized for diagnosing dementia (which assesses orientation, registration, attention and calculation, recall, and language). The study was conducted on 46 cognitively impaired older adults playing a computer game (interactive multimedia internet-based system) for a 12 -week (3 weekly 20-minute sessions) intervention period. Improvements were noted immediately following the intervention $(\mathrm{d}=.32)$, and were present even at a 12-week follow up $(\mathrm{d}=.47)$. There is also research suggesting that engagement in computer games is associated with a higher release of dopamine, a neurotransmitter responsible for learning and reinforcement of behavior ${ }^{34}$ as well as motor rehabilitation benefits..$^{35}$ Additionally, acquiring motivation to play computer games has been associated with an increase in gray matter, which aids brain processes including strategic planning and working memory, as well as motor performance such as navigation strategy. ${ }^{36} \mathrm{~A}$ meta-analysis that covered 36 studies and included 58 effect sizes revealed that, in a population of older adults with MMSE scores over 26, playing video games had a beneficial impact on executive function $(\mathrm{g}=0.76)$ and processing speed $(\mathrm{g}=0.54) .{ }^{37}$ Overall, the available literature points strongly to the cognitive benefits of video game use in older age.

\section{Are skills acquired in video game training transferrable to non-practiced tasks?}

There is ongoing investigation into whether non-practiced skills related to cognitive function can transfer to tasks that were not practiced during video game training. ${ }^{4}$ Generalizability of benefits can become an issue when the skills and abilities gained from using a video game are unique to those tasks practiced in the game. The limitation of generalizability implies that improvements in cognitive abilities acquired from playing video games may not be important in real life. However, several empirical investigations have provided evidence in support of transfer effects. For instance, older adults trained to play commercially available games achieved large gains in outcome variables not directly tied to the games themselves, including improvements in both short-term and long-term memory skills (average $\mathrm{d}=1.17$ ). ${ }^{28}$ Similarly, older individuals playing a commercially available video game (Pac-Man) demonstrated significant improvements in word recall skills- an outcome not directly linked to the video game - compared to a non-video game playing group $(d=0.47) . .^{38}$ Such findings suggest that playing video games can serve as a valuable tool for the improvement of both practiced and not practiced cognitive outcomes and can result in broad-based improvements in cognitive functioning.

Advancements in technology have facilitated the accumulation of a body of literature revealing that older users of video games are 
able to transfer the skills that they learned to domains beyond the ones that they directly practiced. For instance, although multitasking performance scores assessed using a custom-designed 3-D video game (NeuroRacer) deteriorate linearly from 20 to 79 years of age, when older adults age 60 to 85 played an adaptive version of the game (compared to their control groups counterparts), they attained levels that were beyond those of untrained 20 years old individuals, and these gains persisted for 6 months. Electroencephalography showed that age-related deficits decreased after engaging in multitasking training, which led to improvements in control abilities that were not trained, such as working memory and sustained attention. ${ }^{39}$ Additionally, a study required older adults over 65 years of age to maintain information in short term memory by coordinating several cognitive processes corresponding with gaming strategies, as well as utilizing resource management by shifting attention between various stimuli efficiently. The implementation of a real-time strategy game (an adaptation of a commercially available game, Rise of Nations) involving 15 training sessions within 4 to 5 weeks (training session length $=1$ and $1 / 2$ hour) revealed significant differences across several wide-ranging cognitive skills including, but not limited to, reasoning $(d=.55)$, task switching $(d=1.02)$, and working memory $(d=.82) .^{40}$ Furthermore, a meta-analysis that synthesized 20 experimental studies (conducted between 1986 and 2013) revealed that video game training improved a variety of different cognitive functions including global cognition, attention, memory, and reaction time. ${ }^{41}$

It is feasible that the published literature's observed gains in scores on several outcome measures were due, at least in part, to the extensive use of various coordinated cognitive skills. Several studies have demonstrated that engaging in cognitive training, which advances beyond specific skill practicing and focuses more on coordination among complex cognitive strategies, is correlated with broad-based gains in cognitive functioning. These findings may be attributable, at least partially, to the evidence suggesting that other tasks and wideranging gains share common underlying demands with cognitive functions. ${ }^{42}$ It should be noted that not all research in this area uncovered evidence of transfer effects, as the aforementioned body of research is in direct opposition to studies that have reported methodological challenges regarding claims of transferability (see Boot, Blakely, \& Simons $^{43}$ for a review on this topic). Other studies have failed to show that transferability occurs; for instance, when examining the impact of playing video games (in this case Brain Age 2 and Mario Kart) on an array of cognitive outcomes not tied directly to the games in question (e.g., memory and reasoning), researchers were unable to achieve meaningful differences between the video game and control groups with respect to either memory $(d=.01)$ or reasoning $(d=-.12) .{ }^{44}$ Research is needed to test the consistency of the transfer effect across the available literature and to reveal conditions under which transfer is most likely to occur. Given the vastly mixed empirical evidence on this topic, this is an area that requires further investigation. It is possible, for example, that more cognitively demanding video games are more likely to produce transfer effects than less demanding games, but this needs to be verified experimentally.

The outcome measures utilized to assess the impact of cognitive interventions can be categorized by speed of processing and accuracy. Speed of processing measures (e.g., Card Sorting Tasks) typically entail basing scores on the length of time needed to complete a task (usually utilizing a stopwatch or computer device), whereas scores on accuracy measures are usually dependent on the number of correct responses (e.g., MMSE). As previously mentioned, a linear decline in processing speed is most commonly observed as people age. ${ }^{10,45}$ Declines in processing speed often precede cognitive impairments in later life; ${ }^{45}$ as such, video game interventions may have a greater influence on timed measures than on accuracy measures. Relative exposure appears to play a major role in the extent of transferability of skills learned from video game interventions, including processing speed or accuracy measures. Nonetheless, more empirical evidence is warranted prior to drawing any definitive conclusions on this topic.

\section{Conceptualizations of why video game use could improve older adults' cognitive functioning}

Cognitive enrichment hypothesis: The available empirical evidence indicates that there are beneficial effects in implementing video game interventions to improve cognitive functioning in older age. The Cognitive Enrichment Hypothesis ${ }^{4}$ is the notion that malleability of late life-cognition may depend on individual differences. Research on the motivation, feedback, and reinforcement offered by video gaming provides supportive evidence for this notion. ${ }^{46}$ According to this framework; the lower and upper thresholds of cognitive functioning are molded by (a) biological aging and (b) person-specific developmental histories as well as inherited traits. This perspective indicates that the pliability of late life cognition is rooted in the interaction between biological changes caused by the average process of aging and individual discrepancies resulting from experiential and genetic factors. Therefore, the maximum potential for which cognitive intervention could impact cognition is reliant on the upper limits of cognitive functioning of the particular research participant, which is in turn affected by age-related cognitive decline and individual differences. In a study on 35 healthy younger adults (age 20 to 30) and 19 healthy older adults (age 66 to 80), participants were compared on their performance on a word recall task upon engaging in 38 training sessions. The latter were created to improve the encoding and the retrieval of a list of words from memory. Both groups achieved gains in word recall, which suggest the existence of cognitive plasticity within younger and older adults, but there was a sizable difference between age groups $(\mathrm{d}=1.47) .{ }^{47}$ This difference has been reported in several investigations (for more information, see a review by Hertzog and colleagues). ${ }^{4}$ Thus, although some available evidence indicates that older individuals can improve the quality of their cognitive performance via training, not all age-related declines can be fully offset by training. The encouraging news is that, even if older people often are not able to perform at the level of younger adults, they can still improve their cognitive functioning. Supporting the Cognitive Enrichment Hypothesis, ${ }^{4}$ the aforementioned evidence corroborates the notion that video game interventions can enhance cognitive functioning in older age; this is particularly true if the samples in question consist of non-institutionalized individuals with little or no cognitive impairment, as they are typically characterized by higher limits of cognitive functioning.

\section{Other conceptualizations}

Cognitive engagement: Some of the characteristics of the learning experience acquired from video game training are immediate feedback and a high degree of user control that lead participants into an interactive learning experience. Using technology in these conditions can induce positive gains in cognitive skills. ${ }^{48}$

Computer instructions: Allowing game users to choose the pace at which information is delivered can enhance both engagement with the computer-based tool as well as learning. ${ }^{49,50}$ By the same token, 
computer-assisted instructions provide feedback to learners. This feedback is particularly effective when the goals are well-defined; receiving it is highly associated with achieving those goals and is also most valuable when feedback covers achievement/progress regarding current activities, as it can lead learners through activities that are required to achieve specific goals. ${ }^{51}$

Video game instructions: Similar to feedback, video games - in particular those that were created with the intent to enhance cognitive functioning or learning - give users a way to control game pacing. Video games provide an immersive experience, ${ }^{52}$ thus, given their very nature, their use has the potential to increase learning and cognitive engagement, which in turn could increase older adults' chance to attain their maximum cognitive operating level, in line with the aforementioned Cognitive Enrichment Hypothesis. ${ }^{4}$

\section{Video games attitudes and use in relation to self- efficacy and self-esteem}

Since the 1980s and even earlier, research has revealed a negative opinion of video games in older age, ${ }^{53}$ for reasons spanning from lack of interest to lack of perceived need. ${ }^{54}$ Investigations regarding older individuals' attitudes toward video games are scarce, as are those concerning whether such attitudes are related to the efficacy of video game interventions. There is some evidence suggesting a positive link between older adults' attitudes about computer instructions in general and computer technology training. ${ }^{55}$ This pattern has been found in non-video gaming platforms. For example, teaching older adults how to use a digital platform has resulted in greater contact with information and communication technologies, which in turn generated enhanced attitudes towards learning and self-confidence. ${ }^{56}$ Moreover, video game training appears to have a positive effect on older users' attitudes toward video games. ${ }^{54}$

Health status is positively related to attitudes toward computerized technology. ${ }^{57,58}$ Concerning anxious symptomatology, a longitudinal study on youth and older adults showed that intergenerational playing of video games can decrease intergroup anxiety in older age, with older research participants exhibiting lower anxiety levels towards youth. ${ }^{59}$ Additionally, people's beliefs about their capacity to affect their environment are positively related to both their health status and level of cognitive performance. ${ }^{60-64}$ In particular, older adults with strong essentialist beliefs about aging (i.e., activation of negative age stereotypes) have demonstrated weaker memory performance. ${ }^{65}$ Moreover, cognitive performance is highly correlated with individuals' beliefs that cognitive abilities change over time. Indeed, those who believe that these abilities diminish in older age exert less effort towards the maintenance of such abilities and are more likely to view poor performance as an indication of this decline, while those who believe in the malleability of cognition and score higher on selfefficacy are more likely to set high personal goals (including the achievement of milestones in video games) and to commit long-term to achieving these goals, regardless of whether their performance is poor at first. ${ }^{61,66}$

When coupled with corresponding efforts, the aforementioned positive self-perceptions are positively related to improved cognitive performance, ${ }^{60,61}$ with a higher sense of self-efficacy being related to higher levels of cognitive functioning. ${ }^{67,68}$ Furthermore, in an intervention study, engaging in interactive computer-based education enhanced older adults' sense of personal control in long-term care. ${ }^{69}$
Moreover, older adults with a history of hip fracture who underwent virtual reality exposure therapy showed gains in sense of control. ${ }^{70}$ Thus, it is plausible to expect that future video game interventions, based on the newest games, will result in gains in self-efficacy. Self-efficacy and self-esteem appear to be improved by cognitive interventions, perhaps also due to the fact that these two constructs may reflect a higher order common construct, being highly correlated $(r=.60)$, as reported in meta-analytic work covering 75 studies published between 1966 and 2000. ${ }^{71}$

The findings in question suggest that the relationship between self-esteem and self-efficacy is possibly accounted by a single core construct. However, the generalizability of the aforementioned metaanalysis is limited by the fact that the studies covered were conducted mainly on younger adults. Either way, self-efficacy can increase as a function of achieving cognitive gains following video game training. According to the Social Cognitive Theory, ${ }^{72}$ the beliefs that one is capable of achieving success at a task - including playing a video task- including a cognitive process that can increase one's sense of environmental control despite the commonly experienced limitations stemming from a reduction in age-related cognitive abilities. In line with this framework, there is a set of linked constructs, including one's high self-efficacy perceptions, which translate into greater cognitive effort that, conversely, can result in greater feelings of control and cognitive functioning. Cognitive gains have been related to both increased personal control and enhanced self-esteem, as offering older adults a rewarding technology experience in the form of computer training has led to improvements in their self-esteem and sense of control. ${ }^{73}$

\section{Discussion}

The findings of multiple studies suggest that skills obtained from video game use could enhance quality of life for people age 65 or older. Since the 1980s, researchers have found this population to be 5 times more likely to need aid in performing various activities of daily living including meal preparation, self-grooming, money management, and other self-maintenance abilities. ${ }^{74}$ Considering the projected increase in the number and percentage of the population in question, it is likely that a growing number of older adults will experience difficulties in functional abilities. Thus, it is imperative to design technological interventions targeting the enhancement of functional independence and ability to perform activities of daily living in older age. To this end, games utilization can not only enhance cognitive skills, but also be utilized as a way to improve cognitive reserves and subjective well-being. ${ }^{75}$ In turn, this could preserve quality of life in older age and inhibit the onset of dementia (e.g., Alzheimer's disease). This article has provided a concise review of the available literature exclusively in support of the beneficial effects of video game use in older age; this circumscribed scope is a limitation of the article, which was dictated by space limits. Moreover, several video games studied experimentally are now either outdated or not generally accessible to the public. Nonetheless, there could be multiple shared traits among experimentally analyzed video games; those shared characteristics might be generalizable to a large group of comparable video games, some of which are broadly accessible. All things considered, it is too soon to decide whether the benefits achieved from using a particular video game can be generalized to a particular class of video games.

In accordance with the Cognitive Enrichment Hypothesis, ${ }^{4}$ older 
individuals have the plasticity necessary to gain several advantages from playing video games. However, more studies should be conducted to clarify the degree to which the abilities obtained through computer game use in older age impact particular or wide-based cognitive functions. Due to the lack of definite evidence on this topic, consideration should be given to the fact that publicly accessible games that have been tested empirically and have shown promising effects on cognitive functioning in older age could positively impact a greater number of older adults than games that are not available to the public. It is possible that the high amount of feedback and the high level of engagement provided by video games could increase transferability of the skills that older adults gain via using these games; yet, such transferability may depend on the specific nature of each video game and on the level of process coordination that is necessary in order for the older person to adequately play the game. Moreover, in view of the fact that older age often leads to reduced cognitive processing speed, future research on the effect of video games on cognitive functions could cover more thoroughly whether video game interventions have a greater effect on timed than on nontimed cognitive assessments. Overall, there seems to be sufficient empirical evidence to infer that publicly available video games are a conceivably suitable choice for aging individuals who are hoping to improve their cognitive capacities. This could be especially the case for healthy and community-dwelling older adults, who have been the focus of most of the studies reviewed herein.

Additionally, improvements in older adults' cognitive functioning have been associated with psychological benefits such as enhanced self-efficacy. Due to the fact that self-esteem and self-efficacy appear to be improved by participating in cognitive interventions, as described earlier, perhaps engaging in video game use in older age could enhance self-esteem; research is needed in this area. Also, empirical evidence suggests that, with increased exposure to and use of video games, older adults tend to acquire more positive views of this technology. Such technological use could lead to their recognition of the value and advantages of using video games, and this could in turn positively reflect on their self-esteem as they label themselves as video game users; more studies are needed on this topic.

\section{Conclusion}

As a final note, video game activities - especially video game use that also requires the engagement in physical activity - could be conducted within family or geriatric institution settings, with potential additional benefits for older adults that still need to be studied. These activities could strengthen the bond existing among the individuals involved, providing an opportunity for older adults to exercise and interact with their loved ones in an entertaining way. Moreover, gains that are comparable to those described earlier could be achieved if these video game-based activities become part of social gatherings, in addition to fostering social connections and friendships. Based on the aforementioned prior evidence, combining older adults' mental and physical stimulation with social interactions via using video games side by side with others could provide unexplored added bonuses for the enhancement of their quality of life.

\section{Acknowledgements}

This research was supported by a National Institute of General Medical Sciences grant, award number 5SC3GM094075, Luciana
Laganá, Principal Investigator. Additional support for the project was provided by the NIGMS grant \#GM063787 (Research Initiative for Scientific Enhancement) and NIH Building Infrastructure Leading to Diversity (BUILD) grant \#5TL4GM118977. The content of this article does not necessarily represent the official views of the National Institute of General Medical Sciences and is solely the responsibility of the authors.

\section{Conflict of interest}

The author declares no conflict of interest.

\section{References}

1. Clouston SA, Brewster P, Kuh D, et al. The dynamic relationship between physical function and cognition in longitudinal aging cohorts. Epidemiol Rev. 2013;35(1):33-50.

2. Lynch SM. Measurement and prediction of aging anxiety. Res Aging. 2000;22(5):533-558.

3. Palmore E. Ageism comes of age: Review essay. Gerontologist. $2003 ; 43(3): 418-420$

4. Hertzog C, Kramer AF, Wilson RS, et al. Enrichment effects on adult cognitive development: Can the functional capacity of older adults be preserved and enhanced? Psychol Sci Public Interest. 2009;9(1):1-65.

5. Ortman JM, Velkoff VA, Hogan H. An aging nation: the older population in the United States. Washington; DC: US Census Bureau. 2014;25:1140. p.1-28.

6. Alzheimer's Association. Alzheimer's disease facts and figures. Alzheimers Dement. 2017;13(4):325-373.

7. Alzheimer's Association. Alzheimer's disease facts and figures. Alzheimers Dement. 2013;9(2):208-245.

8. Wang M, Gamo NJ, Yang Y, et al. Neuronal basis of age-related working memory decline. Nature. 2011;476(7359):210-213.

9. Broadbent DE, Cooper PF, Fitz Gerald P, et al. Cognitive Failure Questionnaire (CFQ) and its correlates. Brit $J$ Clin Psychol. 1982;21(1):1-16

10. Hills TT, Mata R, Wilke A, et al. Mechanisms of age-related decline in memory search across the adult life span. Dev Psychol. 2013;49(12):2396- 2404

11. Wong JL, Wetterneck C, Klein A. Effects of depressed mood on verbal memory performance versus self-reports of cognitive difficulties. Int $J$ Rehab Health. 2000;5(2):85-97.

12. Cerejeira J, Lagarto L, Mukaetova Ladinska E. Behavioral and psychological symptoms of dementia. Front Neurol. 2012;3:73. p.1-21.

13. Savva GM, Zaccai J, Matthews FE, et al. Prevalence, correlates and course of behavioral and psychological symptoms of dementia in the population. Brit J Psychiat. 2009;194(3):212-219.

14. Bernstein AB, Remsburg RE. Estimated prevalence of people with cognitive impairment: results from nationally representative community and institutional surveys. Gerontologist. 2007;47(3):350-354.

15. Kelly ME, Loughrey D, Lawlor BA, et al. The impact of cognitive training and mental stimulation on cognitive and everyday functioning of healthy older adults:a systematic review and meta-analysis. Ageing Res Rev. 2014;15:28-43.

16. Baltes PB, Lindenberger U, Staudinger UM. Lifespan theory in developmental psychology. 6th ed. In: Damon W, Lerner RM, 
editors. Handbook of child psychology: Theoretical models of human development. Hoboken, Wiley, New Jersey, USA; 2006. p. 569-664.

17. Cheng ST, Chow PK, Song YQ, et al. Mental and physical activities delay cognitive decline in older persons with dementia. Am J Geriat Psychiat. 2014;22(1):63-74.

18. Willis SL, Tennstedt SL, Marsiske M, et al. Long term effects of cognitive training on everyday functional outcomes in older adults. JAMA. 2006;296(23):2805-2814.

19. Rosenberg D, Depp CA, Vahia IV, et al. Exergames for subsyndromal depression in older adults: a pilot study of a novel intervention. $A m \mathrm{~J}$ Geriat Psychiat. 2010;18(3):221-226.

20. Gamberini L, Barresi G, Majer A, et al. A game a day keeps the doctor away: A short review of computer games in mental healthcare. $J$ Cyber Ther Rehabil. 2008;1(2):127-145.

21. Kato PM. Video games in health care: Closing the gap. Rev Gen Psychol. 2010;14(2):113-121.

22. Allaire JC, McLaughlin AC, Trujillo A, et al. Successful aging through digital games: Socio emotional differences between older adult gamers and non-gamers. Comput Hum Behav. 2013;29(4):1302-1306.

23. Primack BA, Carroll MV, McNamara M, et al. Role of video games in improving health-related outcomes: a systematic review. Am J Prev Med. 2012;42(6):630-638.

24. Dustman RE, Emmerson RY, Steinhaus LA, et al. The effects of videogame playing on neuropsychological performance of elderly individuals. J Gerontol. 1992;47(3):168-171.

25. Farris M, Bates R, Resnick H, et al. Evaluation of computer games' impact on cognitively impaired frail elderly. Comput Human Serv. 1994;11(1-2):219-228.

26. Maillot P, Perrot A, Hartley A. Effects of interactive physical-activity video-game training on physical and cognitive function in older adults. Psychol Aging. 2012;27(3):589-600.

27. Shatil E. Does combined cognitive training and physical activity training enhance cognitive abilities more than either alone? A four-condition randomized controlled trial among healthy older adults. Front Aging Neurosci. 2013;26:5-8.

28. Toril P, Reales JM, Mayas J, et al. Video game training enhances visuospatial working memory and episodic memory in older adults. Front Hum Neurosci. 2016;10:206.

29. McDougall S, House B. Brain training in older adults: evidence of transfer to memory span performance and pseudo-Matthew effects. Aging, Neuropsychol Dev Cogn B Aging Neuropsychol Cogn. 2012;19(12):195-221.

30. White N, Cunningham WR. The age comparative construct validity of speeded cognitive factors. Multivar Behav Res. 1987;22(3):249-265.

31. Goldstein JH, Cajko L, Oosterbroek M, et al. Video games and the elderly. Soc Behav Personal. 1997;25(4):345-352.

32. Tarraga L, Boada M, Modinos G, et al. A randomised pilot study to assess the efficacy of an interactive, multimedia tool of cognitive stimulation in Alzheimer's disease. J Neurol Neurosurg Psychiatry. 2006;77(10):11161121.

33. Folstein MF, Folstein SE, Mchugh PR. Mini-mental state: A practical method for grading the cognitive state of patients for the clinicians. $J$ Psychiat Res. 1975;12(3):189-198.

34. Koepp MJ, Gunn RN, Lawrence AD, et al. Evidence for striatal dopamine release during a video game. Nature. 1998;393(6682):266-268.
35. Cameirao MS, Badia SB, Duarte Oller E, et al. The Rehabilitation Gaming System: A virtual reality based system for the evaluation and rehabilitation of motor deficits. Proceedings of Virtual Rehabilitation Venice Italy. 2007;1(1):29-33.

36. Kühn S, Gleich T, Lorenz RC, et al. Playing Super Mario induces structural brain plasticity: gray matter changes resulting from training with a commercial video game. Mol Psychiatr. 2014;19(2):265-271.

37. Zhang F, Kaufman D. Physical and cognitive impacts of digital games on older adults. J Appl Gerontol. 2016;35(11):1189-1210.

38. Secer I, Satyen L. Training skills of divided attention among older adults. JASNH. 2013;9(2):61-78.

39. Anguera JA, Boccanfuso J, Rintoul JL, et al. Video game training enhances cognitive control in older adults. Nature. 2013;501(7465):97101.

40. Basak C, Boot WR, Voss MW, et al. Can training in a real-time strategy videogame attenuate cognitive decline in older adults? Psychol Aging. 2008;23(4):765-777.

41. Toril P, Reales JM, Ballesteros S. Video game training enhances cognition of older adults: a meta-analytic study. Psychol Aging. 2014;29(3):706716.

42. Oei AC, Patterson MD. Enhancing cognition with video games: a multiple game training study. PLoS One. 2013;8(3):e58546.

43. Boot WR, Blakely DP, Simons DJ. Do action video games improve perception and cognition? Front Psychol. 2011;2:226. p.1-6.

44. Boot WR, Champion M, Blakely DP, et al. Video games as a means to reduce age-related cognitive decline: attitudes, compliance, and effectiveness. Front Psychol. 2013;4:31. p.1-9.

45. Salthouse TA. The processing-speed theory of adult age differences in cognition. Psychol Rev. 1996;103(3):403-428.

46. Van Muijden J, Band GP, Hommel B. Online games training aging brains: Limited transfer to cognitive control functions. Front Hum Neurosci. 2012;6:141-154.

47. Baltes PB, Kliegl R. Further testing of limits of cognitive plasticity: Negative age differences in mnemonic skill are robust. Dev Psychol. 1992;28(1):121-125.

48. Sosa GW, Berger DE, Saw AT, et al. Effectiveness of computer-assisted instruction in statistics: A meta-analysis. Rev Educ Res. 2011;81(1):97128.

49. Frederickson N, Reed P, Clifford V. Evaluating web-supported learning versus lecture-based teaching: Quantitative and qualitative perspectives. High Educ. 2005;50(4):645-664.

50. Gonzalez GM, Birch MA. Evaluating the instructional efficacy of computer-mediated interactive media: Comparing three elementary statistics tutorial modules. J Educ Comput Res. 2000;22(4):411-436.

51. Hattie J, Timperley H. The power of feedback. Rev Educ Res. 2007;77(1):81-112.

52. McMahan A. Immersion, engagement, and presence: A Method for analyzing 3-D video games. Inc: Wolf $\mathrm{M}$, Perron $\mathrm{B}$, editors. The video game theory reader. Rutledge, New York, UK; 2003. p. 67-86.

53. McClure RF. Age and video game playing. Percept Motor Skill. 1985;61(1):285-286.

54. Belchior PDC. Cognitive training with video games to improve driving skills and driving safety among older adults [dissertation]. Gainesville: University of Florida, USA; 2007. p. 1-209. 
55. McNeely E. Computer-assisted instruction and the older-adult learner Educ Gerontol. 1991;17(3):229-237.

56. González A, Ramírez MP, Viadel V. Attitudes of the elderly toward information and communications technologies. Educ Gerontol. 2012;38(9):585-594

57. Jay GM, Willis SL. Influence of direct computer experience on older adults' attitudes towards computers. J Gerontol. 1992;47(4):250-257.

58. Menec VH, Chipperfield JG. The interactive effect of perceived control and functional status on health and mortality among young-old and oldold adults. J Gerontol B Psychol Sci. 1997;52(3):118-126.

59. Chua PH, Jung Y, Lwin MO, et al. Let's play together: effects of videogame play on intergenerational perceptions among youth and elderly participants. Comput Hum Behav. 2013;29(6):2303-2311.

60. Bandura A. Regulation of cognitive processes through perceived selfefficacy. Dev Psychol. 1989;25(5):729-735.

61. Bandura A. Perceived self-efficacy in cognitive development and functioning. Educ Psychol. 1993;28(2):117-148.

62. Bandura A, Locke EA. Negative self-efficacy and goal effects revisited J Appl Psychol. 2003;88(1):87-99.

63. Holden G. The relationship of self-efficacy appraisals to subsequent health-related outcomes: A meta-analysis. Soc Work Health Care. 1991;16(1):53-93

64. Multon KD, Brown SD, Lent RW. Relation of self-efficacy beliefs to academic outcomes: A meta-analytic investigation. J Couns Psychol. 1991;38(1):30-38

65. Weiss D. On the inevitability of aging: Essentialist beliefs moderate the impact of negative age stereotypes on older adults' memory performance and physiological reactivity. J Gerontol B Psychol Sci Soc Sci. 2016;1:87. Advance online publication.
66. Wood R, Bandura A. Impact of conceptions of ability on self-regulatory mechanisms and complex decision making. J Pers Soc Psychol. 1989;56(3):407-415.

67. Berry JM. Memory self-efficacy in its social cognitive context. In: Hess TM, Blanchard-Fields F, editors. Social cognition and aging. San Diego, CA: Academic Press, USA; 1999. p. 69-96.

68. Hess TM. Memory and aging in context. Psychol Bull. 2005;131(3):383406.

69. McConatha JT, McConatha, D, Deaner SL, et al. A computer-based intervention for the education and therapy of institutionalized older adults. Educ Gerontol. 1995;21(2):129-138.

70. Giotakos O, Tsirgogianni K, Tarnanas I. A virtual reality exposure therapy (VRET) scenario for the reduction of fear of falling and balance rehabilitation training of elder adults with hip fracture history. Paper presented at the Virtual Rehabilitation Conference, Venice, Italy; 2007.

71. Judge TA, Erez A, Bono JE, et al. Are measures of self-esteem, neuroticism, locus of control, and generalized self-efficacy indicators of a common core construct? J Pers Soc Psychol. 2002;83(3):693-710.

72. Bandura A, Adams NE, Beyer J. Cognitive processing mediating behavioral change. J Pers Soc Psychol. 1977;35(3):125-139.

73. Billipp SH. The psychosocial impact of interactive computer use within a vulnerable elderly population: A report on a randomized prospective trial in home health care setting. Public Health Nurs. 2001;18(2):138-145.

74. Heller PS, Hemming R, Kohnert P, et al. Aging and social expenditure in major industrial countries, 1980-2025. Washington, DC: International Monetary Fund, USA; 1986.

75. Bugos JA, Perlstein WM, McCrae CS, et al. Individualized piano instruction enhances executive functioning and working memory in older adults. Aging Ment Health. 2007;11(4):464-471. 